\title{
Superradiance of quantum dots
}

\author{
MICHAEL SCHEIBNER ${ }^{1 * \dagger}$, THOMAS SCHMIDT ${ }^{1}$, LUKAS WORSCHECH ${ }^{1}$, ALFRED FORCHEL ${ }^{1}$, \\ GERD BACHER ${ }^{2}$, THORSTEN PASSOW ${ }^{3}$ AND DETLEF HOMMEL ${ }^{3}$
}

\author{
${ }^{1}$ Physikalisches Institut, Technische Physik, Universität Würzburg, Am Hubland, 97074 Würzburg, Germany \\ ${ }^{2}$ Werkstoffe der Elektrotechnik, Universität Duisburg-Essen, Bismarckstrasse 81, D-47057 Duisburg, Germany \\ ${ }^{3}$ Institut für Festkörperphysik, Universität Bremen, Otto-Hahn-Allee, D-28359 Bremen, Germany \\ *Present address: Naval Research Laboratory, Washington DC 20375, USA \\ †e-mail: michael.scheibner@physik.uni-wuerzburg.de
}

Published online: 21 January 2007; doi:10.1038/nphys494

In 1954, Dicke pointed out that the description of a spontaneously radiating gas has to include the fact that all atoms or molecules interact with a common radiation field ${ }^{1}$. Consequently, the individual particles may not be considered as independent sources of radiation. In this regard, the question arises of whether quantum dot (QD) systems may also exhibit signatures of cooperative radiation and hence have to be considered as coupled quantum systems. Here, we present experimental evidence for a long-range electromagnetic interaction between laterally arranged QDs. The experimental results suggest that the QDs do not behave like independent objects as long as they form an ensemble of QDs. By removing QDs from the sample, we found that the coupling was reduced. The range of interaction is shown to be at least $150 \mathrm{~nm}$. This may therefore provide a mechanism to couple discrete quantum objects on a large scale.

Quantum dots (QDs) are often treated as independent quantum-mechanical systems. This is based on a comparison between the density with which they are fabricated (for example, $\leq 10^{11} \mathrm{~cm}^{-2}$ by epitaxy) and the wavefunction dimensions of particles such as electrons, holes or excitons, residing in these structures. To make use of these structures in future information processing devices, much effort is being spent on trying to couple at least two of these QDs. So far, the separation between QDs that have been coupled successfully is typically of the order of just a few nanometres ${ }^{2-6}$. The range of a radiative interaction would, by its nature, be of the order of the wavelength of the emitted radiation. Thereby, radiative coupling between QDs becomes considerably interesting for use in quantum-computation schemes ${ }^{7-9}$, especially as laser control of spontaneous collective radiation is predicted to be feasible ${ }^{10}$.

It has been proposed that superradiant effects might also be observable in (inhomogeneously broadened) solid-state systems ${ }^{11-13}$, such as structures that contain many QDs (QD ensembles). To couple QDs through their radiation field, the use of semiconductor microcavities has been suggested ${ }^{7,14}$, and strong coupling between single QDs and the cavity mode has already been demonstrated ${ }^{15,16}$.

We have investigated a sample containing just one single layer of self-assembled CdSe/ZnSe QDs without a microcavity by means of photoluminescence (PL) spectroscopy. The experimental results suggest that the QDs in this sample do not behave like individual independent objects as long as they form an ensemble of QDs. By

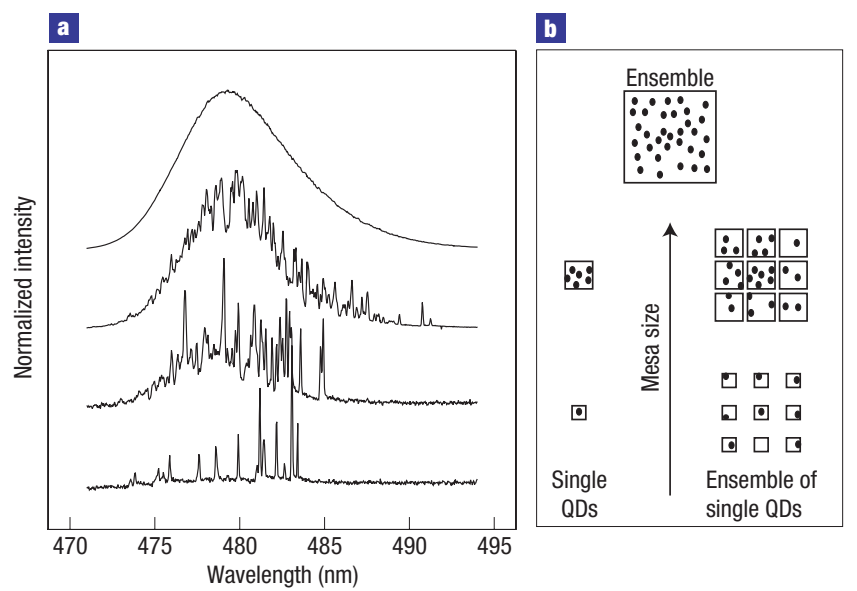

Figure 1 Isolation of QDs from an ensemble. a, Typical PL spectra of single mesas with edge sizes of $25 \mu \mathrm{m}, 1 \mu \mathrm{m}, 350 \mathrm{~nm}$ and $175 \mathrm{~nm}$ (top to bottom). $\mathbf{b}$, Besides single mesas, mesas arranged in grid-like patterns were etched into the sample, to study the radiation of an ensemble of QDs (gas of artificial atoms) versus the radiation of an ensemble of single QDs (diluted gas of artificial atoms). The grid-like patterns serve the purpose of statistical averaging.

successively removing QDs from the sample, we found that the coupling was reduced.

The existence of zero-dimensional states was proved by high spectrally and spatially resolved PL spectroscopy using single nanometre-sized mesas. Successive reduction of the sampling area leads to the appearance of spectrally sharp lines, which resemble the discrete density of states typical for zero-dimensional quantum objects (see Fig. 1 or ref. 17). For this sample, a QD density of $n \leq 10^{11} \mathrm{~cm}^{-2}$ can be deduced, which is equivalent to an average separation between the centres of neighbouring QDs greater than $35 \mathrm{~nm}$. The lateral size of the QDs studied here is 6-10 nm (ref. 18), and the bulk exciton Bohr radius in CdSe $(\mathrm{ZnSe})$ is $5.3 \mathrm{~nm}(9 \mathrm{~nm})$. Thus, they are independent quantum objects in terms of quantummechanical tunnel coupling. In addition, the static dipole moments in the lateral direction (in the QD plane) can be neglected for this type of QDs ${ }^{17}$. 


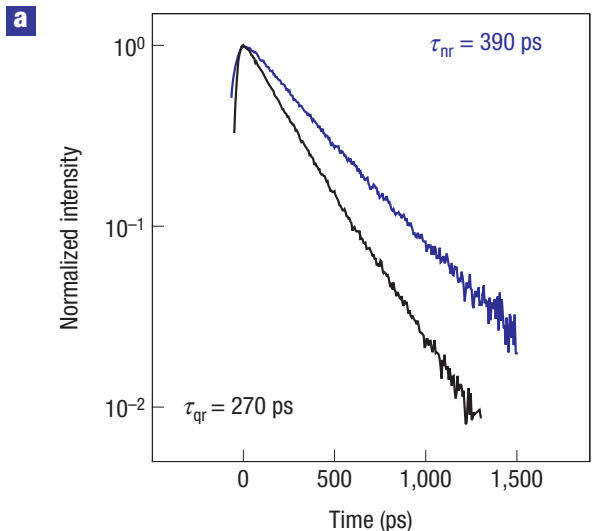

G

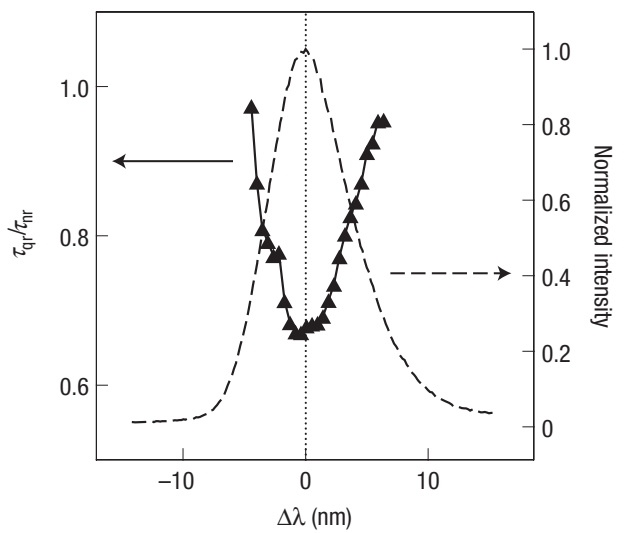

b

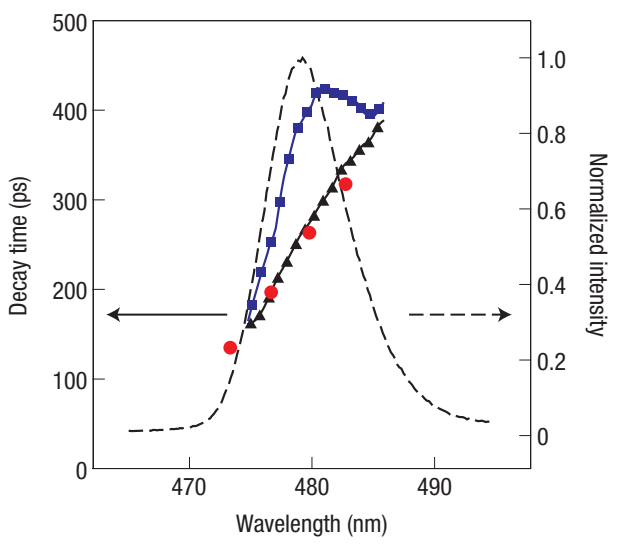

d

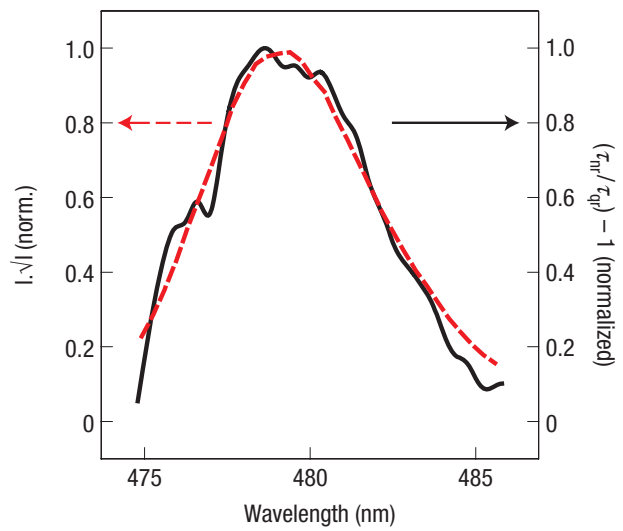

Figure 2 Variation of the PL decay times under different excitation conditions. a, Decay of the $\pi^{\gamma}$ component of the PL signal after non-resonant (blue) and quasi-resonant (black) excitation with $\pi^{\gamma}$ linearly polarized light. b. Spectral dependence of the radiative decay time for non-resonant (blue squares), quasi-resonant (black triangles) and strictly resonant (red circles) excitation. The lines through the data points represent an interpolation of the data points. c, Spectral dependence of the ratio $\tau_{\mathrm{ar}} / \tau_{\mathrm{nr}}$ between quasi-resonant and non-resonant radiative decay time. For comparison, the normalized PL spectrum is included in $\mathbf{b}$ and $\mathbf{c}$ (dashed line). $\mathbf{d}$, Expected (red dashed line) spectral dependence of the change in radiation rate in the case of radiative interaction between the QDs in comparison to the measurement (solid black line). Both curves are normalized.

To test whether such spatially well-separated QDs can interact with each other through their radiation field, it must be shown that changes of the PL decay rate are a function of the number of interacting QDs and their respective separation. This coupling should depend linearly on the number of QDs ${ }^{12}$ and should decrease with the inverse distance between $\mathrm{QDs}^{13}$. Therefore, the PL decay originating from a single layer of CdSe QDs embedded in $\mathrm{ZnSe}$ has been investigated under different conditions. First, the as-grown QD ensemble was studied. After that, mesas of sizes down to a few tens of nanometres were etched into the sample structure, effectively removing some QDs from the sample. For the purpose of statistical averaging, mesas of the same size were arranged in a grid-like pattern with lateral dimensions larger than the size of the laser spot on the sample (see Fig. 1). If there was a radiative interaction between QDs, it would disappear with decreasing mesa size.

Without loss of generality, all of the following experiments were carried out with $\pi^{Y}$ linearly polarized light. It has been shown that the eigenstates of bright excitons in the QDs studied here are predominantly linear superpositions of the two $s$-state exciton spin configurations $| \pm 1\rangle$ (ref. 19). This gives rise to the emission/absorption of linearly polarized light $\pi^{X}$ and $\pi^{Y}$, respectively ${ }^{20,21}$. In addition, the spin relaxation between these two states is known to be at least one order of magnitude slower than the recombination. That means to first approximation these states can be considered to be decoupled, and the transitions of the $\pi^{Y(X)}$ polarized excitons can be treated in terms of two-level systems.

Studying the exciton recombination of the as-grown QD ensemble reveals that the decay rate at the maximum of the PL-spectrum decreases significantly if the excitation is varied between strictly resonant and non-resonant (see Fig. 2a,b). This is in contrast to an expected increase of the decay rate caused by the generation of multi-excitons in the QDs or the population of extended states. A first hint towards identifying the origin of the variation with excitation energy is given by the spectral dependence of the PL decay times (see Fig. 2b). It is well known that the PL decay time increases from the short- to the long-wavelength side of the QD PL spectrum ${ }^{22-24}$. However, the curve shape is different under non-resonant excitation conditions, as compared with strictly or quasi-resonant excitation conditions. Calculating the relative change in decay rate given by the ratio of the PL decay times under quasi-resonant, $\tau_{\mathrm{qr}}$, and non-resonant, $\tau_{\mathrm{nr}}$, conditions $\tau_{\mathrm{qr}} / \tau_{\mathrm{nr}}$ reveals that the strongest change occurs approximately at the wavelength of the maximum of the time-integrated PL signal (see Fig. 2c).

This interesting result may imply that the QDs forming the ensemble are not independent of each other. To first approximation, the PL signal resembles the distribution of 

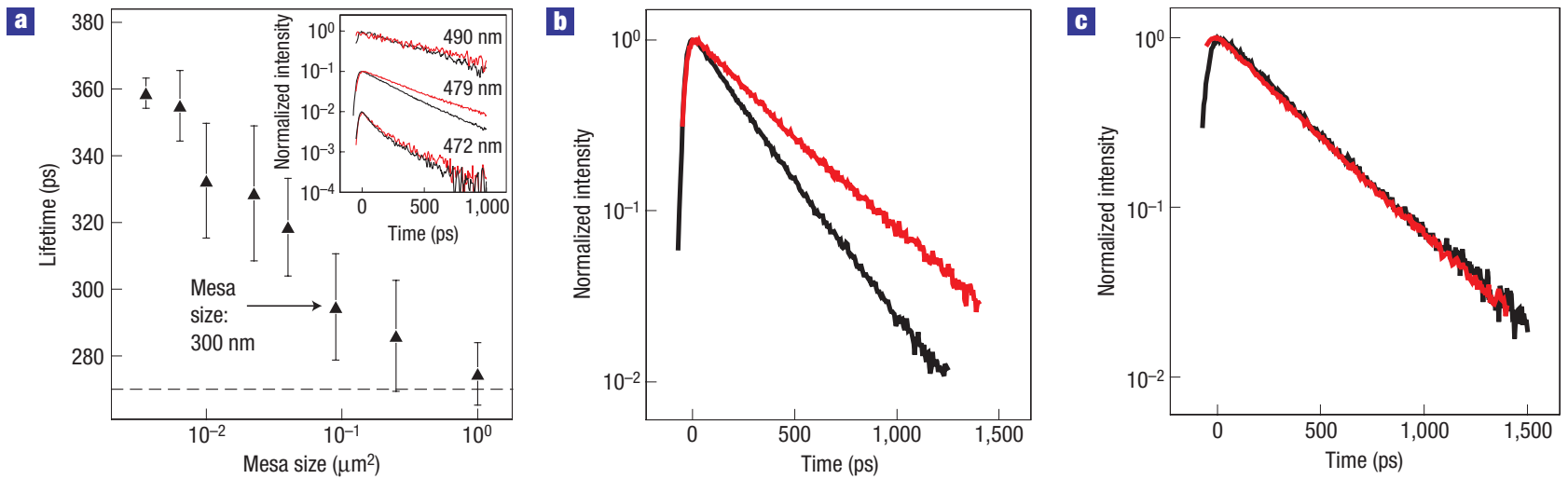

Figure 3 PL decay times for an ensemble of isolated QDs. a, Extracted radiative decay times at the maximum of the PL signal for mesas with different sizes. Every point is the average of three measurements. The error bars are the standard deviation of the average. The dashed line indicates the decay time for the unstructured part of the sample. Inset: Comparison of PL decays at different wavelengths from the unstructured part of the sample (black) and $60 \mathrm{~nm}$ mesas (red). b,c, PL signals from the unstructured part of the sample (black) and $60 \mathrm{~nm}$ mesas (red) under quasi-resonant (b) and non-resonant (c) excitation conditions.

ground-state energies of excitons in the QD ensemble. Consequently, the density of QDs with a specific exciton groundstate energy is highest in the centre of this distribution. It is also obvious that at the centre of the PL signal, the radiation field of the QDs is densest. Considering this, we may return to the question asked in the beginning of this paper. Are QDs that are spatially separated by less than one wavelength of their emitted radiation field really independent of each other?

If this observed change in radiation rate is related to a radiative interaction between QDs it has to scale accordingly. As mentioned above, the fingerprint of a radiative interaction (an altered emission rate) depends linearly on the number of QDs, $N$, and the effect should decrease as $1 / R$, where $R$ is the distance between $\mathrm{QDs}^{12,13}$. This alteration is superimposed on the spectral dependence that is present even in the case of no coupling, $f_{\text {uncoupled }}(\lambda)$. With this, we can write for the emission rate, in the case of coupling (see also Supplementary Information):

$$
\begin{aligned}
f_{\text {coupled }}(\lambda)= & f_{\text {uncoupled }}(\lambda)[1+\Delta(N(\lambda), R(\lambda))] \\
& \text { with } \Delta(N(\lambda), R(\lambda)) \propto \frac{N(\lambda)}{R(\lambda)} .
\end{aligned}
$$

The time-integrated PL spectrum obtained under non-resonant excitation conditions contains information on the number of QDs emitting at a certain wavelength and their respective separation. Therefore, it can be used to calculate the spectral dependence of the change in radiation rate expected in the case of a radiative interaction. With the intensity of the PL signal, $I(\lambda)$, being to first approximation proportional to the number of QDs, $N(\lambda)$, emitting at a wavelength, $\lambda$, it follows that for a fixed area, $A$, (for example, the area of the laser spot) the separation, $R(\lambda)$, between those $N(\lambda)$ QDs relates to the intensity as

$$
I(\lambda) \propto \frac{N(\lambda)}{A} \propto \frac{1}{R(\lambda)^{2}} \Rightarrow \frac{1}{R(\lambda)} \propto \sqrt{I(\lambda)}
$$

and the change in radiation rate due to coupling should vary as

$$
\Delta(N(\lambda), R(\lambda))=\frac{N(\lambda)}{R(\lambda)} \propto I(\lambda) \cdot \sqrt{I(\lambda)}
$$

with the wavelength. This dependence can now be compared with the measured change in PL decay time. For that purpose, the absolute change of the radiation rates $\Delta\left(1 / \tau_{\mathrm{qr}}(\lambda), 1 / \tau_{\mathrm{nr}}(\lambda)\right)$ is calculated, assuming $1 / \tau_{\mathrm{nr}}(\lambda) \hat{=} f_{\text {uncoupled }}(\lambda)$.

$$
\Delta\left(1 / \tau_{\mathrm{qr}}(\lambda), 1 / \tau_{\mathrm{nr}}(\lambda)\right)=\frac{\tau_{\mathrm{nr}}(\lambda)}{\tau_{\mathrm{qr}}(\lambda)}-1 .
$$

The comparison of both dependences reveals a very good correlation (see Fig. 2d). Thus, the observed effect can indeed be attributed to a radiative interaction between the QDs.

Final proof of the suggested mechanism can be given if the effect (a reduced lifetime/increased emission rate at the centre of the PL spectrum) disappears when the interaction between the QDs is inhibited, for example, by removing QDs from the sample. As mentioned above, this can be achieved by etching mesas into the sample structure with nominal sizes down to a few tens of nanometres (see Fig. 1b). The same quasi-resonant excitation conditions as in Fig. 2 were applied. Again, a strong variation of the PL decay time is observed at the maximum of the PL signal, whereas the variation is reduced on either side of the PL spectrum where the density of QDs with the same energy is smaller than at the maximum (see inset in Fig. 3a and Supplementary Information).

Figure $3 \mathrm{~b}$ and $\mathrm{c}$ compare the temporal evolution of the PL signal at the maximum of the PL spectrum from an unstructured part of the sample and mesas of size $60 \mathrm{~nm} \times 60 \mathrm{~nm}$, for quasiresonant as well as non-resonant excitation. The emission rate is clearly reduced for the $60 \mathrm{~nm}$ mesas in the case of quasi-resonant excitation. It should be emphasized that this is in contrast to an expected decrease of the exciton lifetime due to increased nonradiative losses at the generated surfaces. Figure 3 a demonstrates that the radiative lifetime increases steadily with reducing mesa size and therefore the number of QDs that could couple. With the QD density inferred from Fig. 1, a $60 \mathrm{~nm} \times 60 \mathrm{~nm}$ mesa contains less than 4 QDs on average. Therefore, effects due to QD coupling can be assumed to be strongly reduced compared with the unstructured case.

A radiative coupling of QDs implies that the difference of their optical transition energies must be smaller than their homogeneous linewidth. This is similar to the cooperative radiative decay of $N$ periodic quantum wells, for which an $N$ times faster decay compared with a single quantum well was predicted ${ }^{25}$, and experimentally verified with quantum wells that exhibited a non-radiative broadening that was about 20 times larger than the radiative linewidth ${ }^{26}$. Such a situation 
can occur if the QDs are separated energetically not more than their single QD linewidth, which is the homogeneous linewidth broadened by spectral diffusion. For the QDs studied, this typically amounts to a few hundred $\mu \mathrm{eV}$ (ref. 17). This allows the onset of coupling effects to be observed in the range of the studied mesa sizes, as it can be estimated by comparing the probability of finding one QD with that of finding two or more QDs that are separated energetically less than their single QD linewidth within a mesa (see the Supplementary Information).

However, it should be pointed out that even for mesa sizes larger than $300 \mathrm{~nm}$, the radiative decay time continues to decrease. This suggests that placing QDs at an average distance of $150 \mathrm{~nm}$ in the vicinity of other QDs seems to influence the overall radiative emission rate. Therefore, it must be assumed that the range of the interaction between these laterally arranged QDs is at least $150 \mathrm{~nm}$. Note that this coincides with what is expected for a radiative interaction of the QDs as the effective wavelength of the emitted radiation in $\mathrm{ZnSe}$ is about $180 \mathrm{~nm}$.

Carrying out the same experiment with non-resonant excitation exhibits no significant change of the radiative emission rate across the whole range of mesa sizes (see Fig. 3c, $\left.\tau_{\mathrm{nr}}=(375 \pm 20) \mathrm{ps}\right)$. This demonstrates that the observed change under resonant conditions is neither an effect due to a probable alteration in the strain profile which might result from the creation of the mesas, nor does the grid-like pattern of mesas create a new effective dielectric constant with a modified density of photon states. In the case of non-resonant excitation of the as-grown sample, we attribute the decoupling to the capture of unpaired charges and/or the complete loss of polarization during relaxation in the QDs. This reduces the possibility of finding more than one QD with the same ground-state energy and symmetry within the coupling range. In contrast, with (quasi) resonant optical excitation we predominantly populate the superradiant mode of the QD system, because the superradiant mode couples strongly to the optical field, whereas the subradiant mode couples weakly (ideally not at all) to the optical field.

QD systems formed by other materials, for example, InAs/GaAs, should in principle also show radiative coupling ${ }^{13}$. The possibility to observe the onset of coupling effects as was done in this study depends on the peculiar properties of the individual QDs and on the features of the ensemble, such as homogeneity, density, linewidths and emission wavelength, and thus may require a different range of mesa sizes to be chosen.

With $150 \mathrm{~nm}$, the range of interaction observed in this study covers a distance comparable to the dimensions of lithographically well-definable nanostructures. It therefore could be feasible to couple arrays of such well-defined quantum structures. Thereby, this type of coupling could play a significant role in up-scaling novel information processing devices. Further, the optical character of the coupling may also imply its ultrafast application.

\section{METHODS}

The sample was grown by molecular beam epitaxy on a GaAs substrate and a 200-nm-thick GaAs buffer layer. Nominally, 1.3 monolayers of CdSe were deposited on a 50-nm-thick layer of ZnSe. QDs form by self-assembly ${ }^{27}$. Finally, the QDs were capped with a second 25-nm-thick layer of ZnSe.

On one part of the sample, square-shaped mesas were formed by electron beam lithography and dry etching. Single mesas were used to prove the existence of zero-dimensional states. For the purpose of statistical averaging, another part of this sample was patterned with such mesas arranged in arrays of size $100 \mu \mathrm{m} \times 100 \mu \mathrm{m}$ and separations of $1 \mu \mathrm{m}, 2 \mu \mathrm{m}, 5 \mu \mathrm{m}$ and $10 \mu \mathrm{m}$. The mesas were designed with edge lengths ranging from $60 \mathrm{~nm}$ to $1,000 \mathrm{~nm}$, but the actual lengths were about $50 \mathrm{~nm}$ larger.
The sample was investigated using the $363 \mathrm{~nm}$ line of a continuous-wave argon-ion laser as well as a wavelength-tuneable pulsed and frequency-doubled titanium sapphire laser providing $1.5 \mathrm{ps}$ pulses with a repetition rate of $82 \mathrm{MHz}$. The wavelength tuneability of the titanium sapphire laser allowed us to excite the QDs non-resonantly, quasi-resonantly and strictly resonant. The expressions non-resonant, quasi-resonant and strictly resonant refer to excitation above the barrier bandgap energy, excitation between the barrier bandgap energy but above the exciton ground-state energy of the QDs and excitation exactly at the exciton ground-state energy of the QDs, respectively. An achromatic lens was used to focus the laser to a spot of about $60 \mu \mathrm{m}$ in diameter on the sample. Independent of the excitation wavelength, an average excitation intensity of $1 \mathrm{~mW}$ was applied, which did not saturate the QD system, that is, the emitted PL intensity at each wavelength scaled linearly around this excitation power and the average occupancy of QDs with excitons was $\ll 1$. In combination with the argon-ion laser, two additive coupled $0.32 \mathrm{~m}$ monochromators, each equipped with a $1,800 \mathrm{~mm}^{-1}$ line grating, together with a liquid-nitrogen-cooled charge-coupled device camera were used to analyse the PL of the QDs. This set-up had an overall spectral resolution of about $90 \mu \mathrm{eV}$. The emitted PL after excitation with the pulsed laser was spectrally dispersed by a $0.46 \mathrm{~m}$ monochromator equipped with a $300 \mathrm{~mm}^{-1}$ line grating. A streak camera in combination with a Peltier-cooled charge-coupled device camera allowed us to map the temporal evolution of the PL signal. Here, the spectral and temporal resolution were about $1 \mathrm{meV}$ and $<20 \mathrm{ps}$, respectively. The polarization of the laser was adjusted by the use of a Glan-Laser polarizer followed by a Berek polarization compensator. For the polarization-resolved detection of the PL, a dichroic sheet polarizer in combination with a broadband quarter wave plate was used. For all of the experiments, the sample was mounted to the cold-finger of a liquid-helium bath cryostat and kept at a temperature of $2 \mathrm{~K}$.

Received 5 June 2006; accepted 28 November 2006; published 21 January 2007.

References

1. Dicke, R. H. Coherence in spontaneous radiation processes. Phys. Rev. 93, 99-110 (1954).

2. Bayer, M. et al. Coupling and entangling of quantum states in quantum dot molecules. Science $\mathbf{2 9 1}$ 451-453 (2001)

3. Ouyang, M. \& Awschalom, D. D. Coherent spin transfer between molecularly bridged quantum dots. Science 301, 1074-1078 (2003).

4. Krenner, H. J. et al. Direct observation of controlled coupling in an individual quantum dot molecule. Phys. Rev. Lett. 94, 057402 (2005).

5. Petta, J. R. et al. Coherent manipulation of coupled electron spins in semiconductor quantum dots. Science 309, 2180-2184 (2005).

6. Stinaff, E. A. et al. Optical signatures of coupled quantum dots. Science 311, 636-639 (2006)

7. Imamoğlu, A. et al. Quantum information processing using quantum dot spins and cavity QED. Phys. Rev. Lett. 83, 4204-4207 (1999).

8. Biolatti, E., Iotti, R. C., Zanardi, P. \& Rossi, F. Quantum information processing with semiconductor macroatoms. Phys. Rev. Lett. 85, 5647-5650 (2000).

9. Solinas, P., Zanardi, P., Zangh, N. \& Rossi, F. Holonomic quantum gates: A semiconductor-based implementation. Phys. Rev. A 67, 062315 (2003).

10. Macovei, M. \& Keitel, C. H. Laser control of collective spontaneous emission. Phys. Rev. Lett. 91, 123601 (2003).

11. Jodoin, R. \& Mandel, L. Superradiance in an inhomogeneously broadened atomic system. Phys. Rev. A 9, 873-884 (1974).

12. Shahbazyan, T. V., Raikh, M. E. \& Vardeny, Z. V. Mesoscopic cooperative emission from a disordered system. Phys. Rev. B 61, 13266-13276 (2000).

13. Parascandolo, G. \& Savona, V. Long-range radiative interaction between semiconductor quantum dots. Phys. Rev. B 71, 045335 (2005).

14. Temnov, V. V. \& Woggon, U. Superradiance and subradiance in an inhomogeneously broadened ensemble of two-level systems coupled to a low-Q cavity. Phys. Rev. Lett. 95, 243602 (2005).

15. Reithmaier, J. P. et al. Strong coupling in a single quantum dot-semiconductor microcavity system. Nature 432, 197-200 (2004).

16. Yoshie, T. et al. Vacuum Rabi splitting with a single quantum dot in a photonic crystal nanocavity. Nature 432, 200-203 (2004).

17. Seufert, J. et al. Stark effect and polarizability in a single CdSe/ZnSe quantum dot. Appl. Phys. Lett. 79, 1033-1035 (2001).

18. Kümmell, T. et al. Single zero-dimensional excitons in CdSe/ZnSe nanostructures. Appl. Phys. Lett. 73, 3105-3107 (1998).

19. Scheibner, M. et al. Polarization dynamics in self-assembled CdSe/ZnSe quantum dots: The role of excess energy. Phys. Rev. B 67, 153302 (2003).

20. Kulakovskii, V. et al. Fine structure of biexciton emission in symmetric and asymmetric CdSe/ZnSe single quantum dots. Phys. Rev. Lett. 82, 1780-1783 (1999).

21. Puls, J., Rabe, M., Wünsche, H.-J. \& Henneberger, F. Magneto-optical study of the exciton fine structure in self-assembled CdSe quantum dots. Phys. Rev. B 60, R16303-R16306 (1999).

22. Sugawara, $M$. Theory of spontaneous-emission lifetime of Wannier excitons in mesoscopic semiconductor quantum disks. Phys. Rev. B 51, 10743-10754 (1995).

23. Malik, S., Le Ru, E. C., Childs, D. \& Murray, R. Time-resolved studies of annealed InAs/GaAs self-assembled quantum dots. Phys. Rev. B 63, 155313 (2001).

24. Patton, B., Langbein, W. \& Woggon, U. Trion, biexciton, and exciton dynamics in single self-assembled CdSe quantum dots. Phys. Rev. B 68, 125316 (2003).

25. Ivchenko, E. L., Nesvizhskii, A. I. \& Jorda, S. Bragg reflection of light from quantum-well structures. Phys. Solid State 36, 1156-1161 (1994).

26. Hübner, M. et al. Optical lattices achieved by excitons in periodic quantum well structures. Phys. Rev. Lett. 83, 2841-2844 (1999). 


\section{LETTERS}

27. Passow, T. et al. Quantum dot formation by segregation enhanced CdSe reorganization. J. Appl. Phys. 92, 6546-6552 (2002)

\section{Acknowledgements}

We would like to acknowledge financial support by DFG-SFB410, DFG-Ho1388/12 and Ba1422/5 and thank V. D. Kulakowskii for a helpful discussion, M. Emmerling for the sample preparation and

T. L. Reinecke and E. A. Stinaff for valuable comments.

Correspondence and requests for materials should be addressed to M.S

Supplementary Information accompanies this paper on www.nature.com/naturephysics.
Author contributions

T.P. and D.H. fabricated and provided the sample. M.S., L.W. and G.B. conceived the experiments.

M.S. and T.S. carried out the experimental work and data analysis. A.F. provided the experimenta

equipment. M.S., L.W. and G.B. wrote the text with review and input from all other co-authors.

Competing financial interests

The authors declare that they have no competing financial interests.

Reprints and permission information is available online at http://npg.nature.com/reprintsandpermissions/ 